OPEN ACCESS

Edited by:

Wei Xiong,

Tsinghua University, China

Reviewed by:

Jeremy Duncan,

Western Michigan University

United States

Shan Sun,

Fudan University, China

${ }^{*}$ Correspondence:

Jian Zhang

zhj8226@sdu.edu.cn

Jiangang Gao

jggao@sdu.edu.cn

Specialty section:

This article was submitted to

Cell Growth and Division,

a section of the journal

Frontiers in Cell and Developmental

Biology

Received: 21 March 2021

Accepted: 11 May 2021

Published: 04 June 2021

Citation:

Liu Z, Bai X, Wan P, Mo F, Chen G,

Zhang J and Gao J (2021) Targeted Deletion of Loxl3 by Col2a1-Cre

Leads to Progressive Hearing Loss.

Front. Cell Dev. Biol. 9:683495.

doi: 10.3389/fcell.2021.683495

\section{Targeted Deletion of Loxl3 by Col2a1-Cre Leads to Progressive Hearing Loss}

\author{
Ziyi Liu, Xinfeng Bai, Peifeng Wan, Fan Mo, Ge Chen, Jian Zhang* and Jiangang Gao* \\ School of Life Science and Key Laboratory of the Ministry of Education for Experimental Teratology, Shandong University, \\ Jinan, China
}

Collagens are major constituents of the extracellular matrix (ECM) that play an essential role in the structure of the inner ear and provide elasticity and rigidity when the signals of sound are received and transformed into electrical signals. LOXL3 is a member of the lysyl oxidase (LOX) family that are copper-dependent amine oxidases, generating covalent cross-links to stabilize polymeric elastin and collagen fibers in the ECM. Biallelic missense variant of LOXL3 was found in Stickler syndrome with mild conductive hearing loss. However, available information regarding the specific roles of LOXL3 in auditory function is limited. In this study, we showed that the Col2a1-Cre-mediated ablation of Loxl3 in the inner ear can cause progressive hearing loss, degeneration of hair cells and secondary degeneration of spiral ganglion neurons. The abnormal distribution of type II collagen in the spiral ligament and increased inflammatory responses were also found in Col2a1-Lox/3-1- mice. Amino oxidase activity exerts an effect on collagen; thus, Loxl3 deficiency was expected to result in the instability of collagen in the spiral ligament and the basilar membrane, which may interfere with the mechanical properties of the organ of Corti and induce the inflammatory responses that are responsible for the hearing loss. Overall, our findings suggest that Loxl3 may play an essential role in maintaining hearing function.

Keywords: Loxl3, hearing loss, spiral ligament, extracellular matrix, mouse model

\section{INTRODUCTION}

Collagens are major constituents of the extracellular matrix (ECM) and play an essential role in the structure of the inner ear, providing elasticity, and rigidity when the signals of sound are received and transformed into electrical signals. Several types of collagen have been reported in the inner ear, including types I, II, III, IV, V, IX, and XI (Cosgrove et al., 1996; Tsuprun and Santi, 1997). Among these, type II collagen was expressed in the spiral limbus, tectorial membrane (TM), and basilar membrane and was the most abundant component of the spiral ligament (Thalmann, 1993; Dreiling et al., 2002; Husar-Memmer et al., 2013). Furthermore, the TM is composed mostly of the parallel arrangement of type II collagen bundles and associated with other fibrillar and 
nonfibrillar types of collagen, such as Collagen V, IX, and XI (Richardson et al., 1987; Legan et al., 1997; Gavara et al., 2011; Andrade et al., 2016). Both type V collagen and type IV collagen were identified in the strial capillary basement membrane (Cosgrove et al., 1996; Meyer zum Gottesberge and Felix, 2005). More and more mutations of different types of collagen have been identified in both syndromic and non-syndromic hearing loss, such as Stickler syndrome (Vikkula et al., 1995; Richards et al., 1996; Williams et al., 1996), Spondyloepiphyseal dysplasia congenita (Mark et al., 2011; Veeravagu et al., 2013; Xu et al., 2014), Marshall syndrome (Griffith et al., 2000), and Alport syndrome (Mochizuki et al., 1994).

Apart from mutations in genes encoding collagen, a homozygous missense variant in LOXL3 (c.2027G $>$ A, p.Cys676Tyr) was found in two siblings with an autosomal recessive Stickler syndrome, one of the siblings exhibited mild conductive hearing loss (Alzahrani et al., 2015). Stickler syndrome is mostly an autosomal dominant human collagenophathy caused by monoallelic mutations in COL2A1, COL11A2, or COL11A1 (Robin et al., 1993). Stickler syndrome is also inherited in an autosomal recessive manner caused by pathogenic variants in COL9A1, COL9A2, or COL9A3. The symptoms of Stickler syndrome include retinal detachment, auditory dysfunction, hypermobile tympanic membrane, and joint laxity (McAlinden et al., 2008; Acke et al., 2012). LOXL3 is a member of the LOX family that are copper-dependent amine oxidases. All the members can catalyze the oxidative deamination of lysine and hydroxylysine residues, generating covalent cross-links to convert soluble collagen and elastin chains into the insoluble form and stabilize polymeric elastin and collagen fibers in the ECM (Lucero and Kagan, 2006). In the amine oxidase assay, LOXL3 presents amine oxidase activity toward different types of collagen (types I, II, III, IV, VI, VIII, and $\mathrm{X}$ ). Additionally, the amino oxidase activity of LOXL3 could be suppressed by $\beta$-aminopropionitrile $(\beta$-APN), with the inhibition of the formation of covalent cross-links in vivo (Lee and Kim, 2006; Jeong and Kim, 2017). Further studies were performed using several animal models to understand the importance of LOXL3 in development. A zebrafish model with the lack of Loxl3b presented craniofacial defects (van Boxtel et al., 2011). These results were consistent with our previous finding. Our results showed that Loxl3-deficient mice $\left(\right.$ Loxl3 $\left.3^{-/}\right)$exhibited perinatal lethality and severe craniofacial defects, containing palatal cleft and shortened mandible. Additionally, the Loxl3 $3^{-/}$mice showed abnormalities in the cartilage primordia of the thoracic vertebrae (Zhang et al., 2015). Both craniofacial defects and spinal cord deformities are related to the decrease of mature collagen cross-links. However, thus far, the effect of LOXL3 on auditory function has not been studied.

Considering the amino oxidase activity of LOXL3 on collagen, Loxl3 conditional knockout mice in the inner ear were generated by crossing homozygous floxed-Loxl3 mice (Loxl3 ${ }^{\mathrm{f} / \mathrm{f}}$ ) (Zhang et al., 2015) with Col2a1-Cre mice that specifically expressed the Cre recombinase under the Col2al promoter (Sakai et al., 2001). In Col2a1-Cre transgenic mice, the Cre recombinase was first detected at E9-E9.5 in the otic vesicle and notochord
(Sakai et al., 2001). Using the Col2a1-Loxl3 conditional knockout mice, we explored the roles of the Loxl3 gene in the maintenance of auditory function.

\section{RESULTS}

\section{Col2a1-Cre-Mediated Ablation of the Lox/3 Gene and Lox/3 Gene Expression in the Inner Ear}

To investigate the role of Loxl3 in inner ear development, Loxl3 conditional knockout mice were generated with the Cre-loxP system. Homozygous mice (Loxl3 $3^{\mathrm{f} / \mathrm{f}}$ ) carrying the floxed Loxl3 allele were crossed with Col2a1-Cre mice (Figure 1A). The genotypes of the pups were identified by PCR (Figure 1B). We analyzed the expression pattern of Cre recombinase under Col2al using the Rosa26-tdTomato reporter mouse strain (Madisen et al., 2010). At P30, immunofluorescence was performed with sections of the cochlea of the Col2a1-Cre and Rosa26-tdTomato doubly transgenic mice (Figures $\mathbf{1 C}-\mathbf{J}$ ). The Cre recombinase under the Col2al promoter was expressed in the most of spiral limbus (white arrow), all types of fibrocytes in spiral ligament (yellow triangle), basilar membrane (white triangle), and hair cells (blue triangle). The Cre recombinase was expressed sparsely in spiral ganglion cells (yellow arrow) and not expressed in stria vascularis completely. The result of immunofluorescence staining showed that Loxl3 had been inactivated in the spiral limbus (white arrow), basilar membrane (white triangle), outer hair cells (blue triangle), and spiral ligament (yellow triangle). Loxl3 was still expressed in TM (red arrow), part of inner hair cells and spiral ganglion cells (yellow arrow) (Figures 1K-R).

\section{Targeted Inactivation of Loxl3 in the Cochlea by Col2a1-Cre Leads to Progressive Hearing Loss and Degeneration of OHCs and IHCs}

To assess the hearing function of Col2a1-Loxl3-/- mice, auditory brainstem response $(\mathrm{ABR})$ measurements were performed from P30. In broadband click, adult Col2a1Loxl3 $3^{-/-}$mice exhibited no obvious difference in the ABR thresholds at P30. Moreover, the ABR thresholds of the Col2a1Loxl3 $3^{-/-}$mice were increased at $\mathrm{P} 90$ with a sound pressure level (SPL) of averaged $35 \mathrm{~dB}$, whereas the Lox $13^{\mathrm{f} / \mathrm{f}}$ mice showed hearing thresholds of 10-20 dB SPL (Figure 2A). And the data showed a statistically significant increase in the ABR thresholds of the Col2a1-Loxl3 $3^{-/-}$mice. At P150, the Col2a1-Loxl3 $3^{-/-}$ mice exhibited variation of ABR thresholds of $30-70 \mathrm{~dB}$ SPL, and the average of ABR thresholds for broadband click was significantly elevated to approximately $50 \mathrm{~dB}$ compared to $20 \mathrm{~dB}$ in $\operatorname{Loxl3}^{\mathrm{f} / \mathrm{f}}$ mice.

The ABR measurements with frequency-specific pure tone stimuli also showed a progressive increase in the ABR thresholds of Col2a1-Loxl3 ${ }^{-1-}$ mice that were 20-40 dB higher than those in Loxl3 $3^{\mathrm{f} / \mathrm{f}}$ mice until P150 (Figures 2B-D). The results indicate that the targeted inactivation of Loxl3 under Col2al-Cre leads to progressive hearing loss. 
A

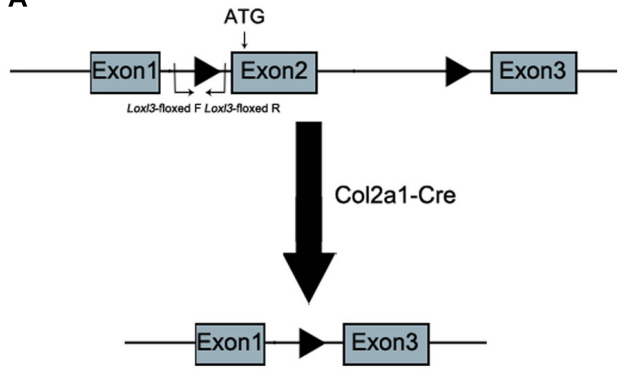

B

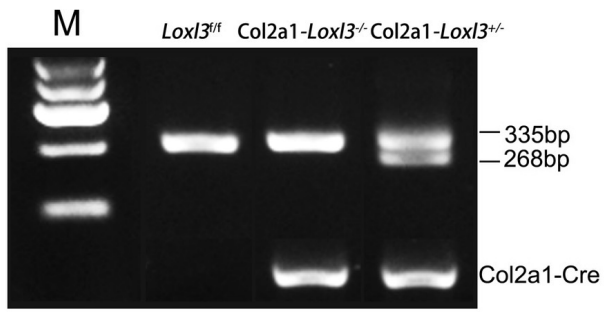

TOMATO/LOXI3/DAPI

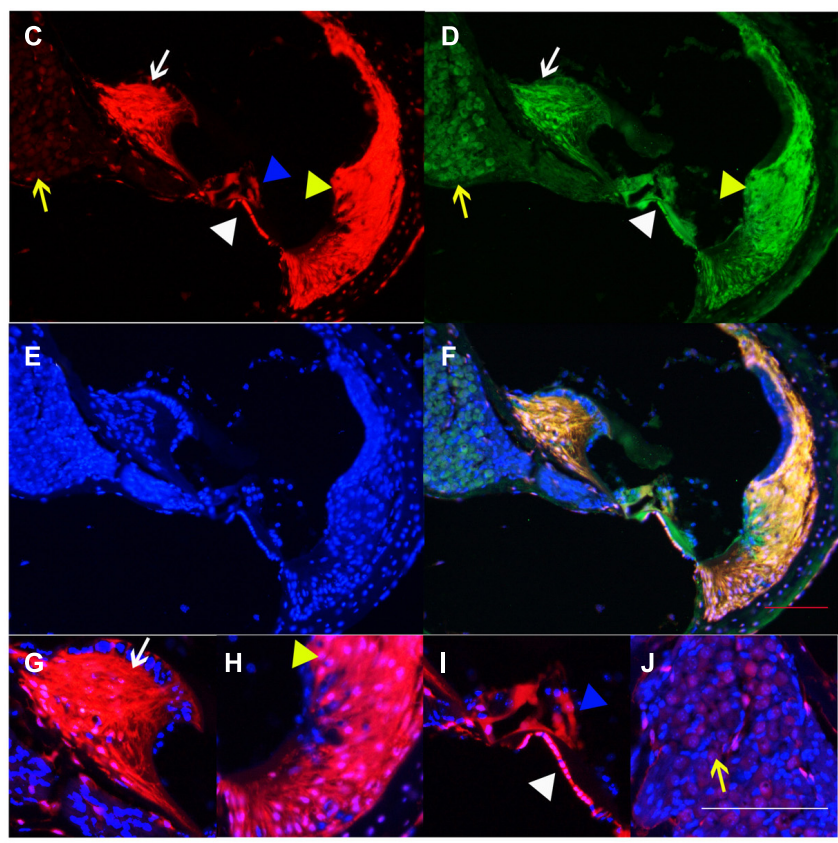

Lox/3f/f
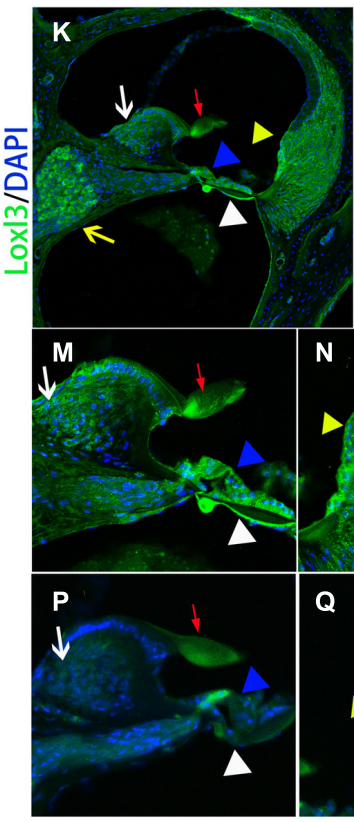

Col2a1-Lox13--

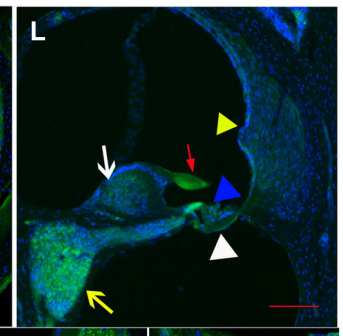

$\mathbf{N}$
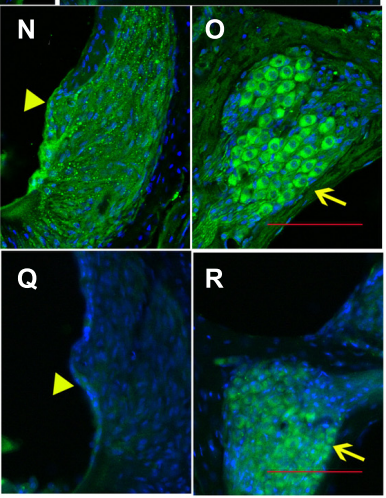

FIGURE 1 | Analysis of Cre recombinase expression in the cochlea of Col2a1-Cre transgenic mice and conditional Loxl3 inactivation in the cochlea of the mice. (A) Schematic diagram of the mice containing floxed Loxl3 alleles crossed with Col2a1-Cre transgenic mice. The deletion of Loxl3 exon 2 leads to deleted ATG start codon for protein translation. (B) Mouse genotyping using PCR analysis. Lane1 wild-type (Lox/3//f) Lane2 homozygous (Col2a1-Lox/3 ${ }^{-/-}$) Lane3 heterozygous $\left(\mathrm{Col} 2 \mathrm{a} 1-\mathrm{Lox}_{3} \mathrm{I}^{-}\right)$mice. (C-F) The Cre recombinase expression under the Col2a1 promoter and expression pattern of Loxl3 in mouse cochlea. The expression of Loxl3 and Cre recombinase was overlapped in the spiral limbus (white arrow), spiral ligament (yellow triangle), basilar membrane (white triangle), and hair cells (blue triangle) The Cre recombinase was expressed sparsely in spiral ganglion cells (yellow arrow). Scale bars: $100 \mu \mathrm{m}$. (G-J) The high magnification of the Cre recombinase expression under the Col2a1 promoter. The expression of Col2a1-Cre recombinase was observed in the most cells of spiral limbus (white arrow), all types of fibrocytes in spiral ligament (yellow triangle), basilar membrane (white triangle), and hair cells (blue triangle). The Cre recombinase was expressed sparsely in spiral ganglion cells (yellow arrow) and not expressed in stria vascularis completely. Scale bars: $100 \mu \mathrm{m}$. (K-L) The targeted inactivation of Loxl3 in Col2a1-Lox/3-/mice. The targeted inactivation of Loxl3 was observed in the spiral limbus (white arrow), spiral ligament (yellow triangle), basilar membrane (white triangle), and hair cells (blue triangle), except spiral ganglion cells (yellow arrow) and tectorial membrane (red arrow). Scale bars: 100 $\mu \mathrm{m}$. (M-0) The high magnification of the Loxl3 expression. The expression of Loxl3 was observed in the spiral limbus (white arrow), basilar membrane (white triangle), hair cells (blue triangle), tectorial membrane (red arrow), spiral ligament (yellow triangle), and spiral ganglion cells (yellow arrow). (P-R) The high magnification of the targeted inactivation of Loxl3 in Col2a1-Lox/3-1- mice. The targeted inactivation of Loxl3 was observed in the spiral limbus (white arrow), basilar membrane (white triangle), outer hair cells (blue triangle), and spiral ligament (yellow triangle). Loxl3 was still expressed in tectorial membrane (red arrow), part of inner hair cells and spiral ganglion cells (yellow arrow). Scale bars: $100 \mu \mathrm{m}$.

To examine the morphology of hair cells in Col2a1$\mathrm{Loxl3}^{-/-}$mice, immunofluorescence assay of cochlear whole mounts was performed and was labeled with phalloidin (green) for stereociliary bundles and Moy7a (red) for hair cell body. Consistent with the results of ABR measurement, few loss of outer hair cells (OHCs) were found at P30 (Figures 3A-F) and approximately 19\% OHC loss was observed in the cochlea basal turn of Col2a1-Loxl3-/mice at P90 (Figures 3G-L). Until P150, significant OHC degeneration was observed in the cochlea basal turn (40\%) 


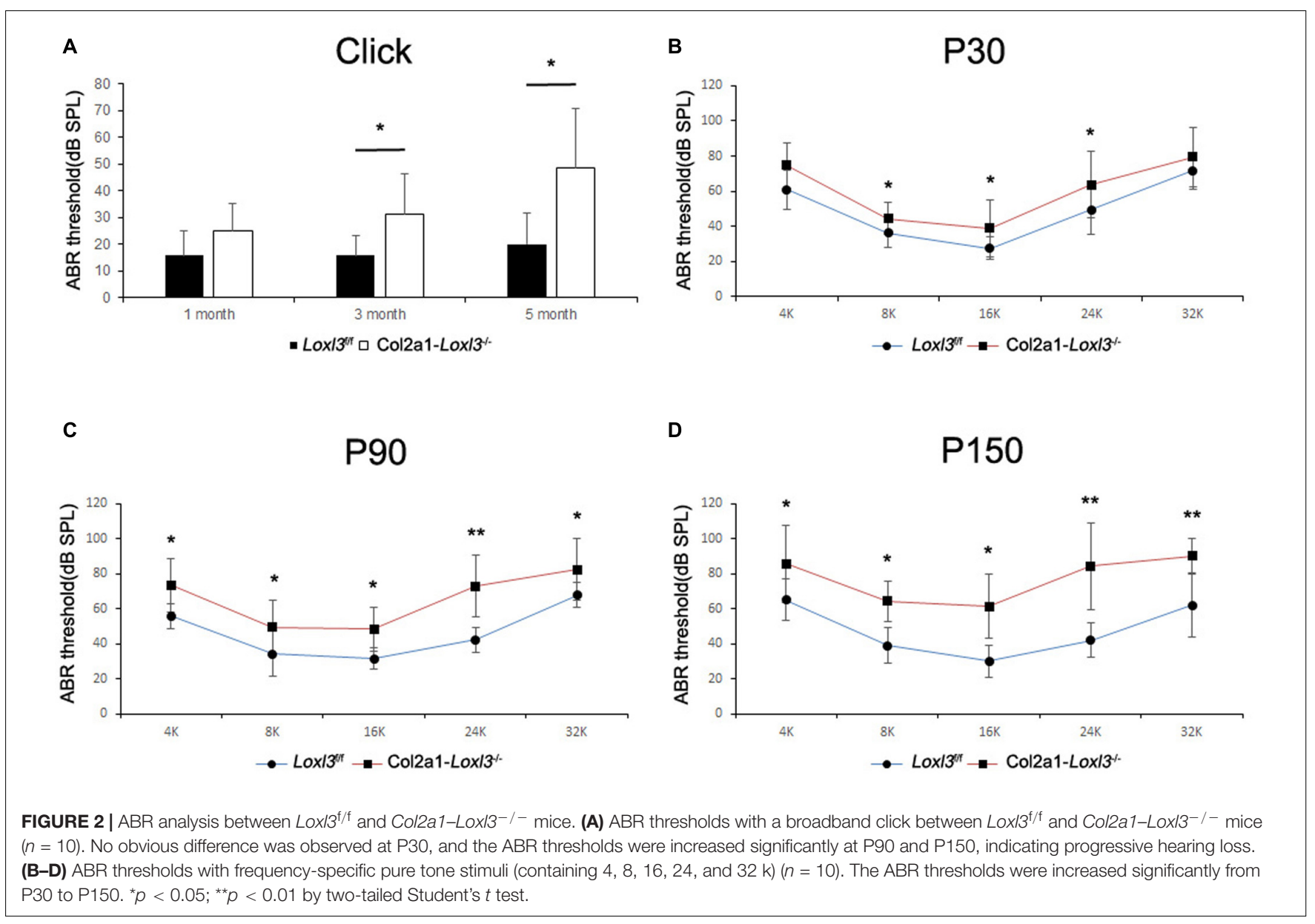

with spotty loss of OHCs in the cochlea middle turn (6\%) of Col2a1-Loxl3 ${ }^{-/-}$mice (Figures 3M-R). The data showed statistically significant differences (Figure 3S). And a small amount of IHCs was also absent in the cochlea basal turn (white asterisk).

\section{Loss of Loxl3 Leads to Impaired Stereocilia of the OHCs and IHCs in the Cochlea}

To examine the stereocilia of the IHCs and OHCs, scanning electron micrography was performed from the apical turn to the basal turn of the cochlea. At P30, the shape and arrangement of the stereocilia were normal in the Col2a1-Loxl3 ${ }^{-/-}$mice (Figures 4A,C), consistent with the results of ABR measurement and immunofluorescence. However, the high magnification of the OHCs in the basal turn of the Col2a1-Loxl3 $3^{-/-}$mice cochlea showed partly stereocilia fusion (Figures 4B,D). Until P150, the stereocilia of the OHCs in the basal turn of the Col2a1-Loxl3 $3^{-/-}$ mice cochlea showed serious impairment and complete fusion, (Figures 4F,H) along with loss of OHCs (Figures $4 \mathbf{E}, \mathbf{G}$ ). The stereocilia of IHCs in Col2a1-Loxl3 ${ }^{-/-}$mice also showed spotty loss (red asterisk). The high magnification of the TM in scanning electron micrograph of Col2a1-Loxl3 ${ }^{-/}$mice showed normal morphology at P150 (Figures 4I-L).

\section{Col2a1-Lox/3-/- Mice Showed Progressive Degeneration of SGNs}

The morphology of the cochlea in the inner ear was investigated by hematoxylin and eosin ( $\mathrm{H} \& \mathrm{E})$ staining. There was no apparent abnormal cochlear morphology in Col2a1-Loxl3 $3^{-/-}$mice at P90 (Figures $\mathbf{5 A}-\mathbf{H}$ ), even in the spiral limbus and spiral ligament, where the Loxl3 was inactivated at P150 (Figures 5IP). The TM of Col2a1-Loxl3 ${ }^{-/-}$mice was also normal at P90 and P150 (Figures 5H,P). However, the spiral ganglion neurons (SGNs) of Col2a1-Loxl3 ${ }^{-/-}$mice appeared degenerated at P150; this may be due to the loss of OHCs (Figure 50). The immunofluorescence microscopy of Tubulin $\beta 3$ (Tuj1), a marker for both types I and II SGNs, was performed (Figures 6A-H). The SGNs data by the serial sections showed that the density of SGNs decreased significantly in Col2a1-Loxl3 $3^{-/-}$mice at P150 (Figure 6I).

\section{Col2a1-Lox/3-/- Mice Showed Abnormal Distribution of Type II Collagen in Spiral Ligament and Increased Inflammatory Responses}

The primary function of Loxl3 is to catalyze the covalent crosslinks of collagen, and type II collagen is the most abundant 


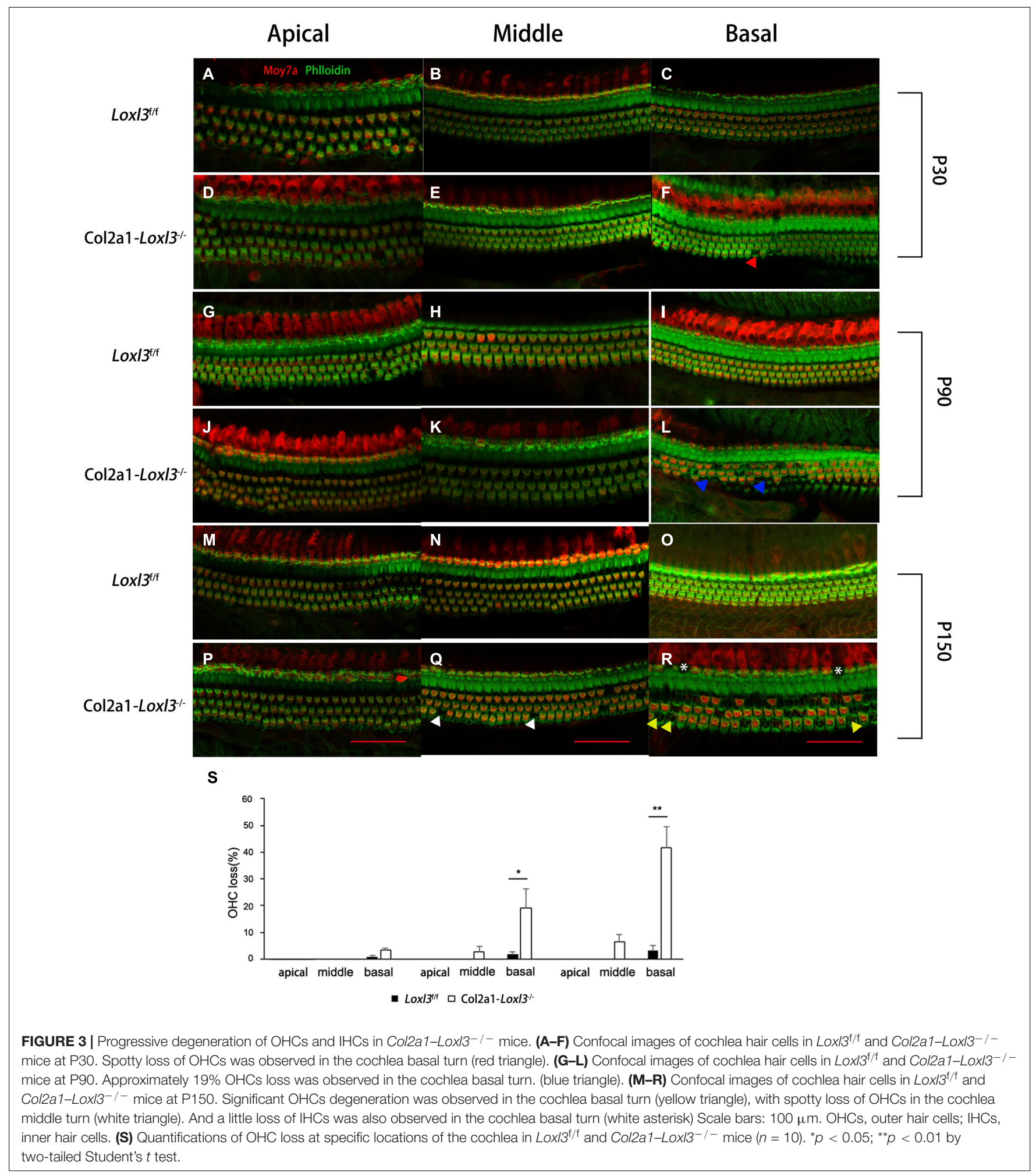

in the whole cochlea (Husar-Memmer et al., 2013). Thus, the distribution of type II collagen was examined in the inner ear. Immunofluorescence staining revealed that the distribution of type II collagen was normal in the TM and spiral limbus of Col2a1-Loxl3 ${ }^{-/-}$mice as compared with that in $\operatorname{Loxl}^{\mathrm{f} / \mathrm{f}}$ mice
(Figures 7A,C). However, the type II collagen fibrils were slightly abnormal in the spiral ligament of Col2a1-Loxl3 ${ }^{-/-}$mice than in those of $L o x l 3^{\mathrm{f} / \mathrm{f}}$ mice. Sparse and curved type II collagen fibrils were observed (Figures 7B,D). Type II collagen plays an essential role in the spiral ligament; thus, the function of the 

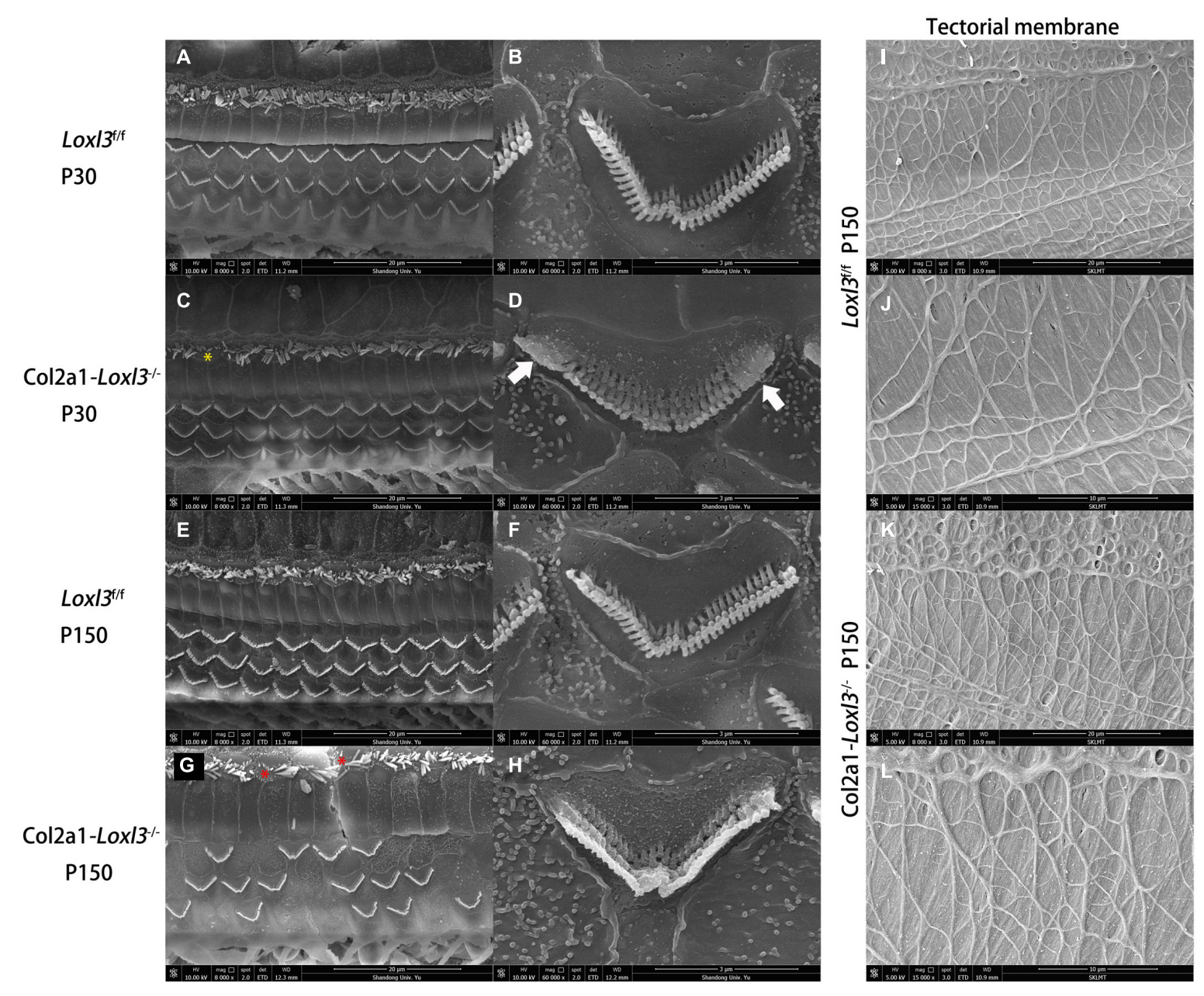

FIGURE 4 | The impaired stereocilia of the outer hair cells and inner hair cells and normal tectorial membrane in Col2a1-Lox/3-/- mice. (A-D) Scanning electron micrograph of hair cell stereocilia in the basal turn of the Lox/3//f and Col2a1-Lox/3-/- cochlea at P30. The magnification of the outer hair cells (OHC) in Col2a1-Lox/3-1- mice showed partial stereocilia fusion (white arrow). And the stereocilia of inner hair cells in Col2a1-Lox/3-/- mice showed spotty loss (yellow asterisk). (E-H) Scanning electron micrograph of hair cell stereocilia in the basal turn of the Lox/3//f and Col2a1-Lox/3-/- cochlea at P150. The stereocilia of the outer hair cells (OHCs) in Col2a1-Lox/3 $3^{-/-}$mice showed serious impairment and complete stereocilia fusion, along with loss of OHCs. And the stereocilia of inner hair cells in Col2a1-Lox/3-/- mice showed spotty loss (red asterisk). (I-L) Scanning electron micrograph of tectorial membrane in the basal turn of the Lox/3 ${ }^{f / f}$ and Col2a1-Lox/3-1- cochlea at P150. The magnification of the tectorial membrane ${ }^{\mathrm{TM}}$ in Col2a1-Lox/3-1- mice showed normal.

spiral ligament fibrocytes may be affected, which are proposed to be associated with the mediation of inflammatory responses to trauma. To detect the inflammatory responses in the cochlea of Col2a1-Loxl3 $3^{-/-}$mice and Loxl3 $3^{\mathrm{f} / \mathrm{f}}$ mice, vascular endothelial growth factor (VEGF), a marker of the inflammatory responses, was analyzed using western blot. The results of western blot analysis showed that the VEGF expression in the cochlea of Col2a1-Loxl3 $3^{-/-}$mice was significantly higher than in those of $\operatorname{Loxl3}^{\mathrm{f} / \mathrm{f}}$ mice at P30-P150, implying the inflammatory responses in the cochlea of Col2a1-Loxl3 $-/-$ mice (Figure 7E).

\section{DISCUSSION}

As demonstrated by the perinatal lethality of the Loxl3-null mice (Zhang et al., 2015), Lox3 plays an critical role in embryonic development. Although a variety novel functions of Loxl3 have been described, the most important biological function of LOXL3 is related to the amino oxidase activity for the crosslinking of collagen.

In this study, we showed that Col2a1-Cre-mediated Loxl3 ablation in the inner ear can cause progressive hearing loss and degeneration of OHCs and IHCs. At P150, the average of ABR thresholds for broadband click in Col2a1-Loxl3 ${ }^{-/-}$mice was significantly elevated to approximately $50 \mathrm{~dB}$ compared with $20 \mathrm{~dB}$ in $\operatorname{Loxl}^{\mathrm{f} / \mathrm{f}}$ mice. Moreover, no apparent morphologic abnormality was found at the light microscopic level in whole mounts and cross-sections of inner ears. However, the SGNs in Col2a1-Loxl3 ${ }^{-/}$mice appeared degenerated at P150; this may be caused by the loss of OHCs. An irreversible damage to cochlear hair cells is often followed by secondary degeneration of SGNs (Dodson and Mohuiddin, 2000; Xu et al., 2021). The 


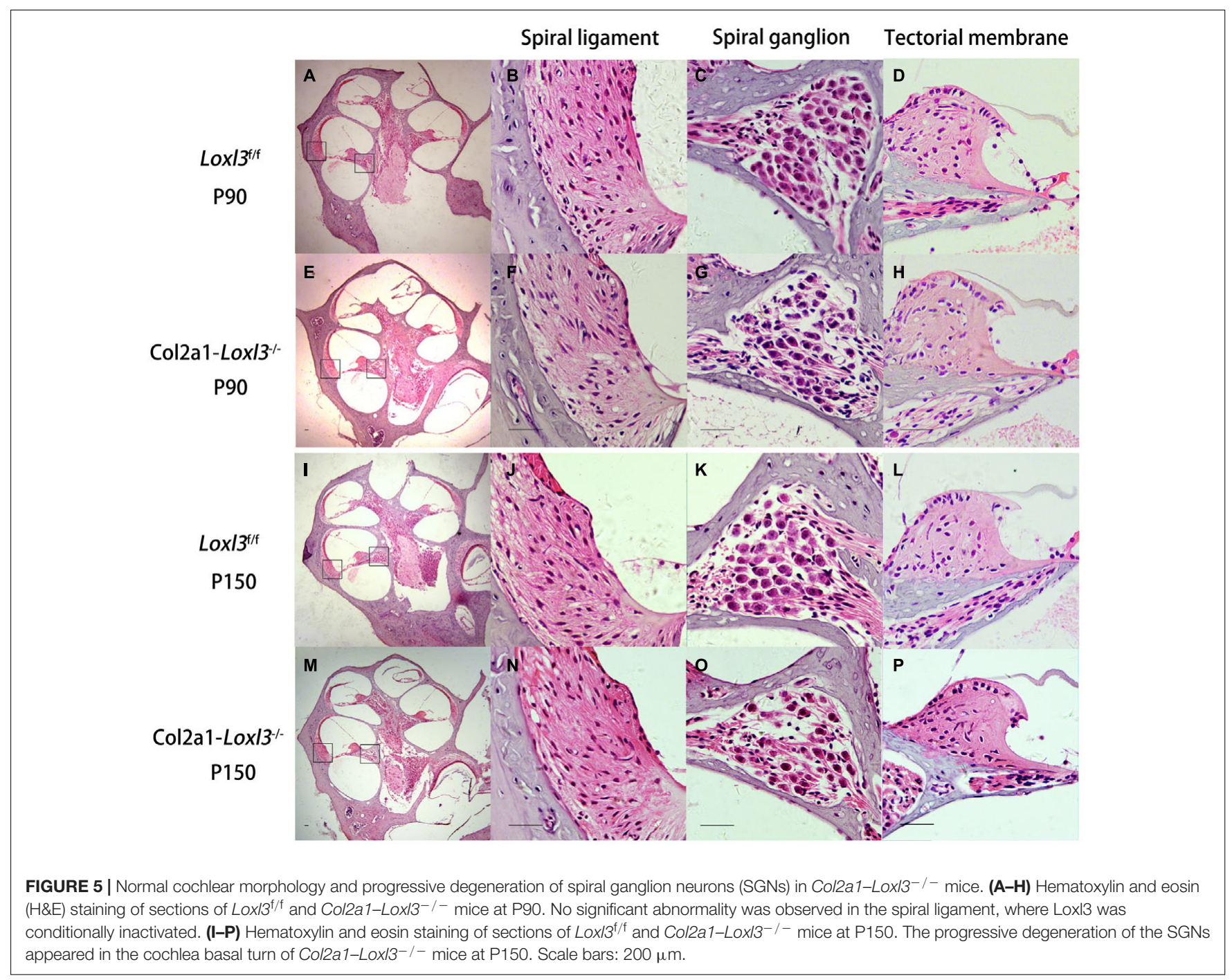

degeneration of SGNs usually starts with the loss of synaptic terminals followed by the disintegration of cell bodies (Pan et al., 2011; Shibata et al., 2011).

In the previous study, we crossed the Loxl ${ }^{\mathrm{f} / \mathrm{f}}$ mice with Atoh1-Cre mice that primarily express the Cre recombinase in hair cells, and the mice with deletion of Loxl3 mediated by Atoh1 promotor did not show hearing loss and degeneration of OHCs (data not shown), suggesting that the precise ablation of Loxl3 in hair cells was not able to affect the function of OHCs. The results of this study showed that the expression of Cre recombinase in Col2a1-Cre transgenic mice overlapped with the expression pattern of Loxl3, including the spiral limbus, basilar membrane, and spiral ligament; hence, the primary function of Loxl3 in the inner ear may be to catalyze the cross-links of collagen. Moreover, the deficiency of Loxl3 was expected to result in collagen instability that leads to the semblable phenotypes in Col2a1-Loxl3 ${ }^{-/-}$mice and several mouse models with mutations of collagen, such as progressive hearing loss in type IX collagen knockout mice (Suzuki et al., 2005).
Among the several types of collagen expressed in the inner ear, type II collagen is the most abundant throughout the cochlea; it is encoded by Col2a1 and comprises three identical alpha1(II) chains (Husar-Memmer et al., 2013). The results of Type II collagen immunofluorescence showed that the deposition of type II collagen was basically in the TM, spiral limbus and spiral ligament. The type II collagen fibrils were regular in the TM and spiral limbus, consistent with the results of H\&E staining. However, the type II collagen fibrils were sparse and curved in the spiral ligament of Col2a1-Loxl3 $3^{-/-}$mice compared with those

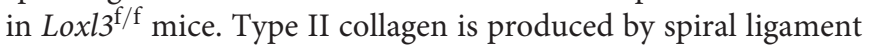
fibrocytes, and the abnormal staining could be a sign of pathology (Tsuprun and Santi, 1999; Buckiova et al., 2006). Type II collagen is also the main component of the spiral ligament where collagen fibrils provide stability and strength to the ECM and maintain the integrity of the ion transport systems (Spicer and Schulte, 1991). As shown by the results, the expression of Loxl3 was basically deleted in the spiral ligament; which might affect the crosslink of collagen and led to abnormal type II collagen fibrils. Then the function of spiral ligament was affected. 


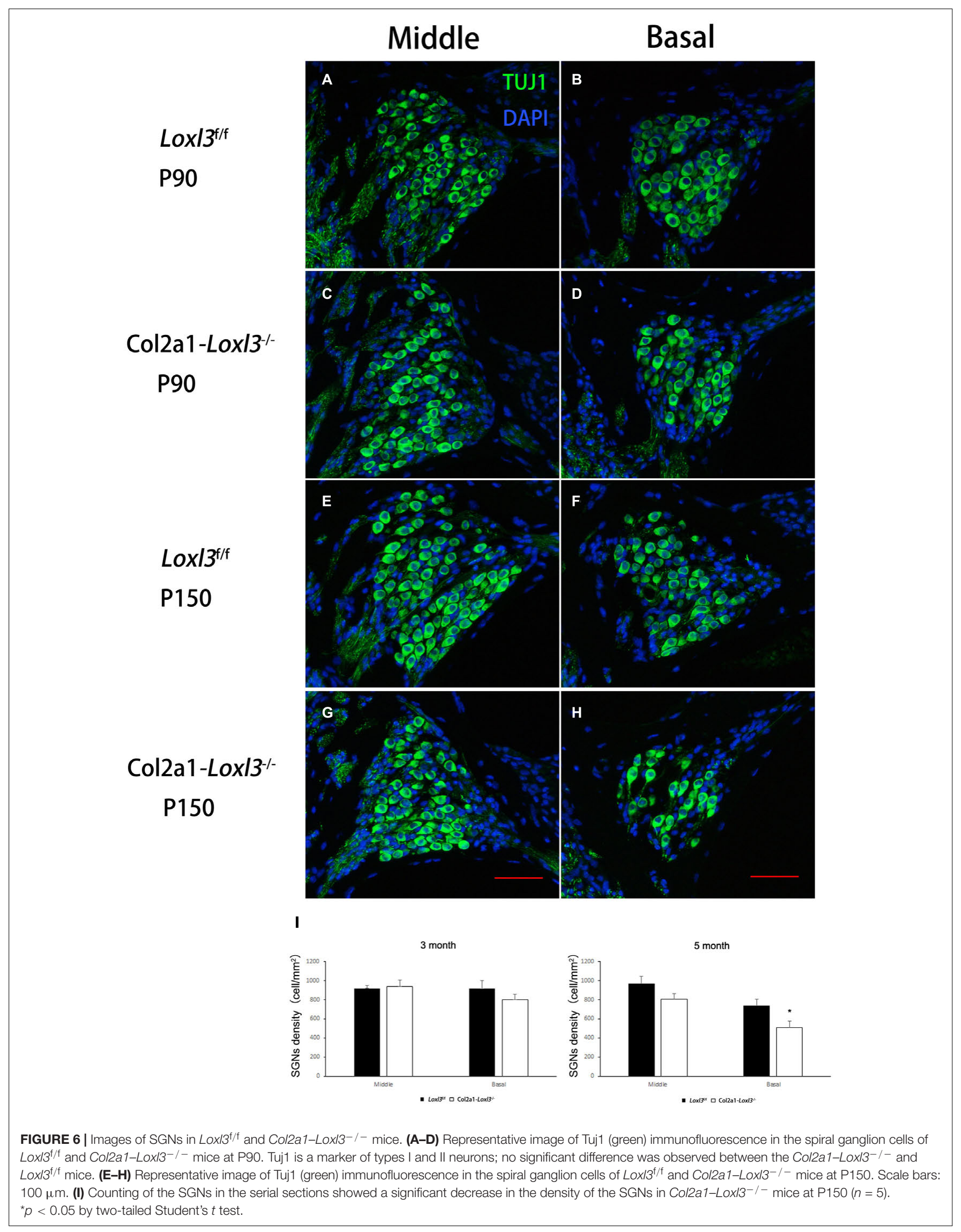




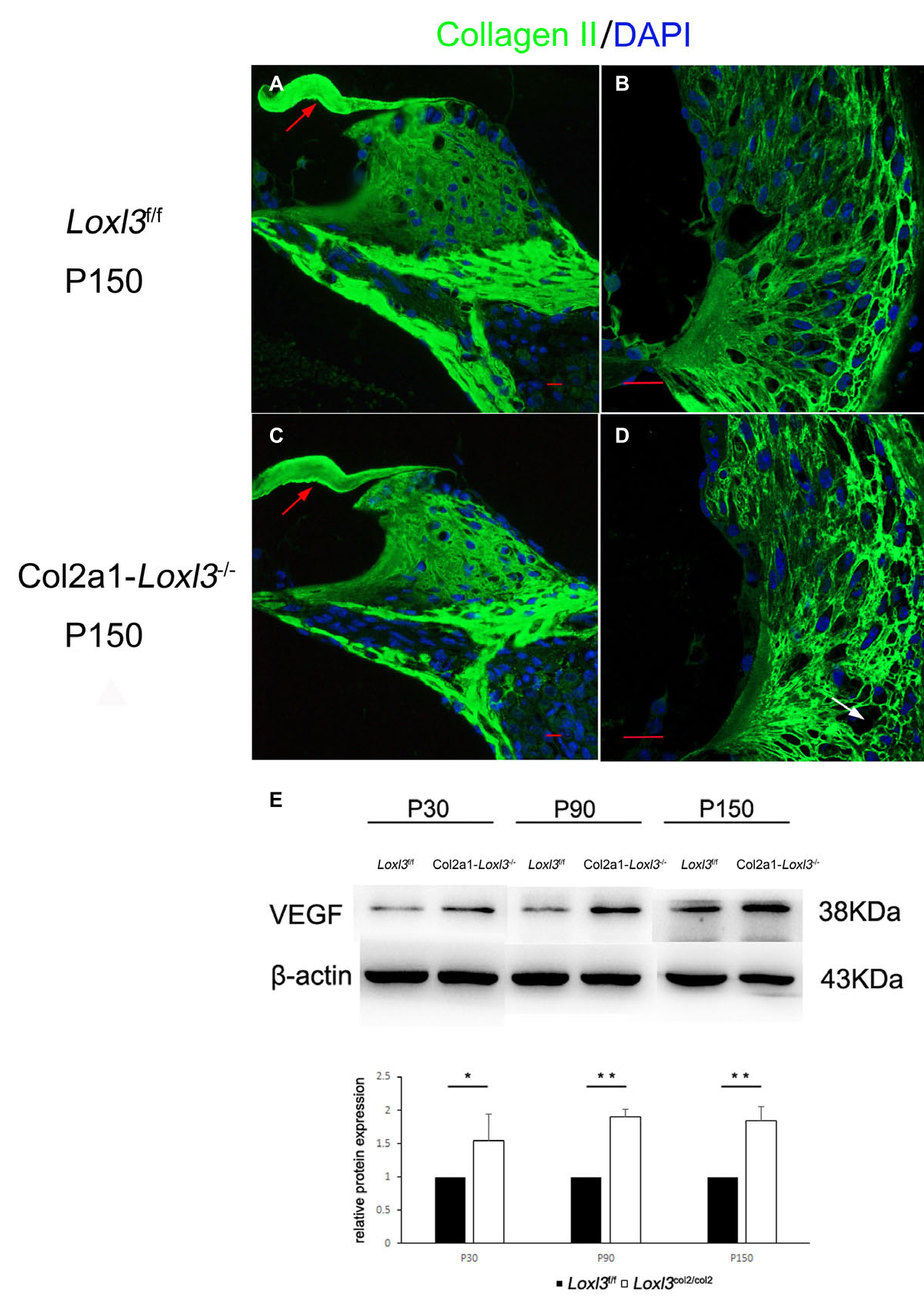

FIGURE 7 | Distribution of Type II collagen in the spiral ligament and the expression of vascular endothelial growth factor (VEGF) in the cochlea of Lox/3 ${ }^{f / f}$ and Col2a1-Lox/3 ${ }^{-/-}$mice. (A,C) Representative image of type II collagen (green) immunofluorescence in the spiral limbus and the tectorial membrane of $L$ ox/ $3^{f / f}$ and Col2a1-Lox/3 ${ }^{-/-}$mice at P150. The deposition of type II collagen was normal in the tectorial membrane of Col2a $1-L o x / 3^{-/-}$mice than in those of Lox/3f/f mice (red arrow). (B,D) Representative image of type II collagen (green) immunofluorescence in the spiral ligament of Lox/3/f and Col2a1-Lox/3-/- mice at P150. The deposition of type II collagen was slightly abnormal in the spiral ligament of Col2a1-Lox/3 $-/-$ mice than in those of $L o x / 3^{f / f} \mathrm{mice}(\mathrm{white}$ arrow). Scale bars: $20 \mu \mathrm{m}$. (E) Western blot analysis of VEGF expression in the cochlea of Col2a1-Lox/3-/- and Lox/3 ${ }^{f / f}$ mice at P30-P150. The results of western blot analysis showed that the VEGF expression was significantly increased in the cochlea of Col2a1-Lox/3-/- mice than in those of Lox/3 ${ }^{\mathrm{f} / \mathrm{f}}$ mice at P30-P150 $\left(n=5 ;{ }^{*} p<0.05 ;{ }^{* \star} p<0.01\right)$.

Western blot analysis showed that the VEGF expression was significantly increased in the cochlea of Col2a1-Loxl3 ${ }^{-/-}$mice than in those of Loxl3 ${ }^{\mathrm{f} / \mathrm{f}}$ mice at P30-P150. It had been reported that VEGF expression was upregulated at the onset of cochlear noise-induced damage. The VEGF upregulation was observed in the stria vascularis, spiral ligament and spiral ganglion and the expression of VEGF-receptors was normal (Picciotti et al., 2006). Therefore, these pathological phenotypes of Col2a1-Loxl3 ${ }^{-/-}$ mice were similar to the cochlear damage induced by noise or ototoxic antibiotics (Jiang et al., 2017; Sha and Schacht, 2017). 
Moreover, the death of hair cells and SGNs induced by noise or ototoxic antibiotics was mostly associated with macrophage recruitment and cochlear inflammation (Kaur et al., 2018; He et al., 2020). In noise-induced hearing loss, the damage to fibrocytes of the spiral ligament is likely to lead to changes in cytokines or chemokines such as TNF-a, IL-1b, IL-6, and Icam-1 (Fujioka et al., 2006, 2014; Tornabene et al., 2006). The cochlear spiral ligament is a connective tissue that is suggested to play an essential role in the pathophysiology of different etiologies of hearing loss. The spiral ligament is composed of several specialized fibrocytes that are proposed to play different roles in fluid homeostasis, the mediation of inflammatory responses to trauma, and the fine tuning of cochlear mechanics (Hequembourg and Liberman, 2001; Wangemann, 2006; Ohlemiller, 2009). VEGF could induce vascular neogenesis and was produced by spiral ligament fibrocytes stimulated by IL-1L or TNF-K. These inflammatory response mediators together might induce inflammatory cell movement that would prolong the inflammatory response and impair hair cells (Yoshida et al., 1999). Thus, the abnormal type II collagen fibrils caused by inactivated Loxl3 may affect the function of spiral ligament and lead to inflammatory response in inner ear of Col2a1-Loxl3 ${ }^{-/-}$mice, resulting in the loss of hair cells and secondary degeneration of SGNs. Then the Col2a1-Loxl3 ${ }^{-/-}$mice showed progressive hearing loss.

Type II collagen has also been detected in the basilar membrane, overlapping with the deletion of Loxl3 in Col2a1Loxl3 $3^{-/}$mice. Both sensory hair cells and supporting cells are localized on the basilar membrane; Deiters' and pillar cells are essential for constituting a cochlear amplifier, providing necessary structural support for the hair cells (Cosgrove et al., 1996; Meyer zum Gottesberge and Felix, 2005). It has been discovered that the deficiency of one collagen-binding receptor (DDR1) in mice leads to both the abnormal morphology of the supporting cells and the alteration of OHCs. It is noteworthy that the DDR1-null mice also showed ABR thresholds shift at P60, explaining that the variation in intracellular electron density of Deiters' cells led to the reduced anchorage to the basilar membrane and impairment in OHCs (Meyer zum Gottesberge et al., 2008). In Col2a1-Loxl3 ${ }^{-/-}$mice, the structural abnormality of the basilar membrane and Deiters' cells lead to the degeneration of OHCs, and the ultrastructural features of the basilar membrane are expected to be observed in further investigation.

In conclusion, we have demonstrated that Loxl3 plays an essential role in the maintenance of the inner ear function; the results may provide more explanation for the pathology of Stickler syndrome caused by homozygous mutations in LOXL3.

\section{MATERIALS AND METHODS}

\section{Animals}

Rosa26-tdTomato (No. 007914) and Col2a1-Cre (No. 003554) mice were obtained from the Jackson Laboratory. Mice homozygous for floxed Loxl3 exon2 were crossed with Col2a1-Cre mice. The following primers were used for mouse genotyping: Loxl3-floxed F (5'-CCCTTCCTGTCACA TCCTGT- $3^{\prime}$ ) and Loxl3-floxed R (5'-AACAGGCACAGCCCT AGAGA-3 $\left.{ }^{\prime}\right)$ for the floxed Loxl3 allele and Cre-F (5'-GCATC GACCGGTAATGCAGGC-3') and Cre-R (5'-AGGGTCCAG CCCGAGCTACTT-3') for specific Col2a1-Cre.

\section{Auditory Brainstem Response Measurement}

Auditory brainstem response was performed in a soundproof room. The mice were anesthetized with pentobarbital sodium (50 mg/kg body weight) via intraperitoneal injection. Three electrodes were inserted subcutaneously in the mice through the cranial vertex, the external ear, and the back near the tail. Brand click and tone burst stimuli of $4,8,16$, 24 , and $32 \mathrm{kHz}$ were generated, and signals of responses were recorded using a Tucker Davis Technologies (TDT, United States) workstation running SigGen32 software (TDT, United States). The ABR thresholds were determined using the lowest sound intensity at which the first wave could be elicited clearly.

\section{Immunostaining Analysis}

The cochlea was fixed in $4 \%$ formaldehyde at $4^{\circ} \mathrm{C}$ overnight and decalcified in $10 \%$ EDTA at room temperature for at least $24 \mathrm{~h}$. For sectioning, the cochleae were dehydrated with $15 \%$ sucrose for $2 \mathrm{~h}$ and later in $30 \%$ sucrose overnight at $4^{\circ} \mathrm{C}$. Samples were embedded in Tissue-Tek OCT compound and frozen at $-20^{\circ} \mathrm{C}$ and then sectioned into 9 - $\mu \mathrm{m}$-thick slices. For whole-mount immunostaining, the sensory epithelium in the cochlea was isolated and divided into several parts of apical, middle, and basal turns. Then, the sections or whole cochlea samples were permeabilized with $0.5 \%$ Triton X-100 in PBS at room temperature for $15 \mathrm{~min}$, washed in PBS thrice, and then blocked in $10 \%$ goat serum in $\mathrm{PBS}$ at $374^{\circ} \mathrm{C}$ for $30 \mathrm{~min}$. The samples were incubated with a primary antibody at $4^{\circ} \mathrm{C}$ overnight. After washing with PBS, further incubation with a secondary antibody (goat anti-rabbit Alexa-488 or Alexa-568, 1:500; Invitrogen) diluted in $\mathrm{PBS}$ at $37^{\circ} \mathrm{C}$ for $1 \mathrm{~h}$ was performed, followed by Alexa Fluor 488-conjugated phalloidin (Sigma-Aldrich, United States) at $37^{\circ} \mathrm{C}$ for $30 \mathrm{~min}$ and DAPI at RT for $5 \mathrm{~min}$. Immunofluorescence images were collected using an LSM 880 confocal microscope (Zeiss). The primary antibodies included rabbit anti-LOXL3 (1:200, American Research Products, United States), rabbit anti-myosin VIIa (1:200, Cell Signaling, United States), and rabbit anti-Tuj1 (1:400, Cell Signaling, United States).

\section{Scanning Electron Microscopy}

The inner ears of the mice were dissected and fixed with 2.5\% glutaraldehyde overnight at $4^{\circ} \mathrm{C}$ and decalcified in 10\% EDTA at room temperature for at least $24 \mathrm{~h}$. The sensory epithelium in the cochlea was isolated and post-fixed in $1 \%$ osmium tetroxide for $2 \mathrm{~h}$. The samples were dehydrated through a graded ethanol series and critically point dried. The samples were then mounted and sputter-coated with gold. Thereafter, stereociliary bundles 
and TM were examined using a Hitachi S-4800 Field Emission scanning electron microscope.

\section{Histology Analysis}

The cochlea samples were fixed and decalcified using a similar procedure as described in the immunostaining assay; then, they were dehydrated with an ethanol series ranging from 30 to $100 \%$ and embedded in paraffin to then be sectioned at a thickness of $5 \mu \mathrm{m}$ and stained using $\mathrm{H} \& \mathrm{E}$.

\section{Western Blot}

$\operatorname{Loxl}_{3}{ }^{\mathrm{f} / \mathrm{f}}$ and Col2a1-Loxl3 $3^{-/-}$mice were sacrificed via cervical dislocation, and their cochleae were dissected. Cochleae proteins were incubated in cell lysis buffer (P0013, Beyotime) and extracted using a homogenizer. Western blot analyses were performed as described previously (Hou et al., 2014). The following primary antibodies were used: anti-VEGFA polyclonal antibody (rabbit, 1:400, ABclonal) and anti- $\beta$-actin polyclonal antibody (rabbit, 1:3,000, Proteintech). All data are presented as mean \pm standard error of the mean, and data analyses were performed using the Image $J$ software. Student's $t$ test was used for single-factor experiments involving two groups. The significance level was set to $p<0.05$ for all statistical analyses.

\section{Statistical Analysis}

Data were expressed as the mean \pm SD and indicated from at least three independent experiments. Statistical analysis of the data was performed using a two-tailed-distribution Student's $t$ test or one-way ANOVA using GraphPad Software. For all tests, a value of $p<0.05$ was considered to be statistically significant.

\section{DATA AVAILABILITY STATEMENT}

The original contributions presented in the study are included in the article/supplementary material, further inquiries can be directed to the corresponding authors.

\section{REFERENCES}

Acke, F. R., Dhooge, I. J., Malfait, F., and De Leenheer, E. M. (2012). Hearing impairment in Stickler syndrome: a systematic review. Orphanet J. Rare Dis. 7:84. doi: 10.1186/1750-1172-7-84

Alzahrani, F., Al Hazzaa, S. A., Tayeb, H., and Alkuraya, F. S. (2015). LOXL3, encoding lysyl oxidase-like 3 , is mutated in a family with autosomal recessive Stickler syndrome. Hum. Genet. 134, 451-453. doi: 10.1007/s00439-015-1531-z

Andrade, L. R., Salles, F. T., Grati, M., Manor, U., and Kachar, B. (2016). Tectorins crosslink type II collagen fibrils and connect the tectorial membrane to the spiral limbus. J. Struct. Biol. 194, 139-146. doi: 10.1016/j.jsb.2016.01.006

Buckiova, D., Popelar, J., and Syka, J. (2006). Collagen changes in the cochlea of aged Fischer 344 rats. Exp. Gerontol. 41, 296-302. doi: 10.1016/j.exger.2005.11.010

Cosgrove, D., Samuelson, G., and Pinnt, J. (1996). Immunohistochemical localization of basement membrane collagens and associated proteins in the murine cochlea. Hear. Res. 97, 54-65. doi: 10.1016/s0378-5955(96)80007-4

Dodson, H. C., and Mohuiddin, A. (2000). Response of spiral ganglion neurones to cochlear hair cell destruction in the guinea pig. J. Neurocytol. 29, 525-537.

\section{ETHICS STATEMENT}

The animal study was reviewed and approved by Ethics Committee of Shandong University. All experimental procedures about animals were approved by Ethics Committee of Shandong University. Animal management was performed strictly in accordance with the standards of the Animal Ethics Committee of Shandong University (Permit Number: ECAESDUSM 20123004).

\section{AUTHOR CONTRIBUTIONS}

ZL: writing-original draft, investigation, software, and writingreview and editing. XB: writing-original draft, investigation, and software. PW, FM and GC: investigation. JZ: funding acquisition and writing-review and editing. JG: writing-review and editing, funding acquisition, supervision, resources, project, and administration. All authors contributed to the article and approved the submitted version.

\section{FUNDING}

This work was supported by grants from the Natural Science Foundation of China (No. 81670943), the National Basic Research Program of China (No. 2014CB541703), the Fundamental Research Fund of Shandong University (Grant No. 2017JC038), the Shandong Provincial Natural Science Foundation of China (Grant No. ZR2017MC028), and the National Key Research and Developmental Program of China (Grant No. 2018YFC1003602-3).

\section{ACKNOWLEDGMENTS}

We thank Sen Wang, Haiyan Yu, Xiaomin Zhao, and Yuyu Guo from State Key Laboratory of Microbial Technology, Shandong University for assistance in microimaging of SEM analysis.

Dreiling, F. J., Henson, M. M., and Henson, O. W. Jr. (2002). The presence and arrangement of type II collagen in the basilar membrane. Hear. Res. 166, 166-180. doi: 10.1016/s0378-5955(02)00314-3

Fujioka, M., Kanzaki, S., Okano, H. J., Masuda, M., Ogawa, K., and Okano, H. (2006). Proinflammatory cytokines expression in noise-induced damaged cochlea. J. Neurosci. Res. 83, 575-583. doi: 10.1002/jnr.20764

Fujioka, M., Okamoto, Y., Shinden, S., Okano, H. J., Okano, H., Ogawa, K., et al. (2014). Pharmacological inhibition of cochlear mitochondrial respiratory chain induces secondary inflammation in the lateral wall: a potential therapeutic target for sensorineural hearing loss. PLoS One 9:e90089. doi: 10.1371/journal. pone.0090089

Gavara, N., Manoussaki, D., and Chadwick, R. S. (2011). Auditory mechanics of the tectorial membrane and the cochlear spiral. Curr. Opin. Otolaryngol. Head Neck Surg. 19, 382-387. doi: 10.1097/moo.0b013e32834a 5 bc9

Griffith, A. J., Gebarski, S. S., Shepard, N. T., and Kileny, P. R. (2000). Audiovestibular phenotype associated with a COL11A1 mutation in Marshall syndrome. Arch. Otolaryngol. Head Neck Surg. 126, 891-894. doi: 10.1001/ archotol.126.7.891 
He, W., Yu, J., Sun, Y., and Kong, W. (2020). Macrophages in noise-exposed cochlea: changes, regulation and the potential role. Aging Dis. 11, 191-199. doi: 10.14336/ad.2019.0723

Hequembourg, S., and Liberman, M. C. (2001). Spiral ligament pathology: a major aspect of age-related cochlear degeneration in C57BL/6 mice. J. Assoc. Res. Otolaryngol. 2, 118-129. doi: 10.1007/s101620010075

Hou, C., Ding, L., Zhang, J., Jin, Y., Sun, C., Li, Z., et al. (2014). Abnormal cerebellar development and Purkinje cell defects in Lgl1-Pax2 conditional knockout mice. Dev. Biol. 395, 167-181. doi: 10.1016/j.ydbio.2014.07.007

Husar-Memmer, E., Ekici, A., Al Kaissi, A., Sticht, H., Manger, B., Schett, G., et al. (2013). Premature osteoarthritis as presenting sign of Type II collagenopathy: a Case report and literature review. Semin. Arthritis Rheu. 42, 355-360. doi: 10.1016/j.semarthrit.2012.05.002

Jeong, C., and Kim, Y. (2017). LOXL3-sv2, a novel variant of human lysyl oxidaselike 3 (LOXL3), functions as an amine oxidase. Int. J. Mol. Med. 39, 719-724. doi: $10.3892 /$ ijmm.2017.2862

Jiang, M. Y., Karasawa, T., and Steyger, P. S. (2017). Aminoglycoside-induced cochleotoxicity: a review. Front. Cell Neurosci. 11:308. doi: 10.3389/fncel.2017. 00308

Kaur, T., Ohlemiller, K. K., and Warchol, M. E. (2018). Genetic disruption of fractalkine signaling leads to enhanced loss of cochlear afferents following ototoxic or acoustic injury. J. Comp. Neurol. 526, 824-835. doi: 10.1002/cne. 24369

Lee, J. E., and Kim, Y. (2006). A tissue-specific variant of the human lysyl oxidaselike protein 3 (LOXL3) functions as an amine oxidase with substrate specificity. J. Biol. Chem. 281, 37282-37290. doi: 10.1074/jbc.m600977200

Legan, P. K., Rau, A., Keen, J. N., and Richardson, G. P. (1997). The mouse tectorins. modular matrix proteins of the inner ear homologous to components of the sperm-egg adhesion system. J. Biol. Chem. 272, 8791-8801.

Lucero, H. A., and Kagan, H. M. (2006). Lysyl oxidase: an oxidative enzyme and effector of cell function. Cell Mol. Life Sci. 63, 2304-2316. doi: 10.1007/s00018006-6149-9

Madisen, L., Zwingman, T. A., Sunkin, S. M., Oh, S. W., Zariwala, H. A., Gu, H., et al. (2010). A robust and high-throughput Cre reporting and characterization system for the whole mouse brain. Nat. Neurosci. 13, 133-140. doi: 10.1038/nn. 2467

Mark, P. R., Torres-Martinez, W., Lachman, R. S., and Weaver, D. D. (2011). Association of a p.Pro786Leu variant in COL2A1 with mild spondyloepiphyseal dysplasia congenita in a three-generation family. Am. J. Med. Genet. A. 155A, 174-179. doi: 10.1002/ajmg.a.33762

McAlinden, A., Majava, M., Bishop, P. N., Perveen, R., Black, G. C., Pierpont, M. E., et al. (2008). Missense and nonsense mutations in the alternatively-spliced exon 2 of COL2A1 cause the ocular variant of Stickler syndrome. Hum. Mutat. 29, 83-90. doi: 10.1002/humu.20603

Meyer zum Gottesberge, A. M., and Felix, H. (2005). Abnormal basement membrane in the inner ear and the kidney of the Mpv17-/- mouse strain: ultrastructural and immunohistochemical investigations. Histochem. Cell Biol. 124, 507-516. doi: 10.1007/s00418-005-0027-7

Meyer zum Gottesberge, A. M., Gross, O., Becker-Lendzian, U., Massing, T., and Vogel, W. F. (2008). Inner ear defects and hearing loss in mice lacking the collagen receptor DDR1. Lab. Invest. 88, 27-37. doi: 10.1038/labinvest. 3700692

Mochizuki, T., Lemmink, H. H., Mariyama, M., Antignac, C., Gubler, M. C., Pirson, Y., et al. (1994). Identification of mutations in the alpha 3(IV) and alpha 4(IV) collagen genes in autosomal recessive Alport syndrome. Nat. Genet. 8, 77-81. doi: 10.1038/ng0994-77

Ohlemiller, K. K. (2009). Mechanisms and genes in human strial presbycusis from animal models. Brain Res. 1277, 70-83. doi: 10.1016/j.brainres.2009. 02.079

Pan, N., Jahan, I., Kersigo, J., Kopecky, B., Santi, P., Johnson, S., et al. (2011). Conditional deletion of Atoh1 using Pax2-Cre results in viable mice without differentiated cochlear hair cells that have lost most of the organ of Corti. Hear. Res. 275, 66-80. doi: 10.1016/j.heares.2010.12.002

Picciotti, P. M., Fetoni, A. R., Paludetti, G., Wolf, F. I., Torsello, A., Troiani, D., et al. (2006). Vascular endothelial growth factor (VEGF) expression in noise-induced hearing loss. Hear. Res. 214, 76-83. doi: 10.1016/j.heares.2006. 02.004
Richards, A. J., Yates, J. R. W., Williams, R., Payne, S. J., Pope, F. M., Scott, J. D., et al. (1996). A family with Stickler syndrome type 2 has a mutation in the COL11A1 gene resulting in the substitution of glycine 97 by valine in alpha 1 (XI) collagen. Hum. Mol. Genet. 5, 1339-1343. doi: 10.1093/hmg/5.9.1339

Richardson, G. P., Russell, I. J., Duance, V. C., and Bailey, A. J. (1987). Polypeptide composition of the mammalian tectorial membrane. Hear. Res. 25, 45-60. doi: 10.1016/0378-5955(87)90078-5

Robin, N. H., Moran, R. T., and Ala-Kokko, L. (1993). "Stickler syndrome," in GeneReviews [Internet], Edn, eds M. P. Adam, H. H. Ardinger, R. A. Pagon, S. E. Wallace, L. J. H. Bean, and K. Stephens et al. (Seattle (WA): University of Washington, Seattle.).

Sakai, K., Hiripi, L., Glumoff, V., Brandau, O., Eerola, R., Vuorio, E., et al. (2001). Stage-and tissue-specific expression of a Col2al-Cre fusion gene in transgenic mice. Matrix Biol. 19, 761-767. doi: 10.1016/s0945-053x(00)00122-0

Sha, S. H., and Schacht, J. (2017). Emerging therapeutic interventions against noiseinduced hearing loss. Expert Opin. Investig. Drugs 26, 85-96. doi: 10.1080/ 13543784.2017.1269171

Shibata, S. B., Budenz, C. L., Bowling, S. A., Pfingst, B. E., and Raphael, Y. (2011). Nerve maintenance and regeneration in the damaged cochlea. Hear. Res. 281, 56-64. doi: 10.1016/j.heares.2011.04.019

Spicer, S. S., and Schulte, B. A. (1991). Differentiation of inner-ear fibrocytes according to their iron-transport related activity. Hear. Res. 56, 53-64. doi: 10.1016/0378-5955(91)90153-Z

Suzuki, N., Asamura, K., Kikuchi, Y., Takumi, Y., Abe, S., Imamura, Y., et al. (2005). Type IX collagen knock-out mouse shows progressive hearing loss. Neurosci. Res. 51, 293-298. doi: 10.1016/j.neures.2004.12.001

Thalmann, I. (1993). Collagen of accessory structures of organ of Corti. Connect. Tissue Res. 29, 191-201. doi: 10.3109/03008209309016826

Tornabene, S. V., Sato, K., Pham, L., Billings, P., and Keithley, E. M. (2006). Immune cell recruitment following acoustic trauma. Hear. Res. 222, 115-124. doi: 10.1016/j.heares.2006.09.004

Tsuprun, V., and Santi, P. (1997). Ultrastructural organization of proteoglycans and fibrillar matrix of the tectorial membrane. Hear. Res. 110, 107-118. doi: 10.1016/s0378-5955(97)00068-3

Tsuprun, V., and Santi, P. (1999). Ultrastructure and immunohistochemical identification of the extracellular matrix of the chinchilla cochlea. Hear. Res. 129, 35-49. doi: 10.1016/s0378-5955(98)00219-6

van Boxtel, A. L., Gansner, J. M., Hakvoort, H. W. J., Snell, H., Legler, J., and Gitlin, J. D. (2011). Lysyl oxidase-like $3 \mathrm{~b}$ is critical for cartilage maturation during zebrafish craniofacial development. Matrix Biol. 30, 178-187. doi: 10.1016/j. matbio.2010.12.002

Veeravagu, A., Lad, S. P., Camara-Quintana, J. Q., Jiang, B., and Shuer, L. (2013). Neurosurgical interventions for spondyloepiphyseal dysplasia congenita: clinical presentation and assessment of the literature. World Neurosurg. 80, e1-e8.

Vikkula, M., Mariman, E. C., Lui, V. C., Zhidkova, N. I., Tiller, G. E., Goldring, M. B., et al. (1995). Autosomal dominant and recessive osteochondrodysplasias associated with the COL11A2 locus. Cell 80, 431-437. doi: 10.1016/00928674(95)90493-x

Wangemann, P. (2006). Supporting sensory transduction: cochlear fluid homeostasis and the endocochlear potential. J. Physiol. 576(Pt 1), 11-21. doi: 10.1113/jphysiol.2006.112888

Williams, C. J., Ganguly, A., Considine, E., McCarron, S., Prockop, D. J., WalshVockley, C., et al. (1996). A-2-> G transition at the $3^{\prime}$ acceptor splice site of IVS17 characterizes the COL2A1 gene mutation in the original Stickler syndrome kindred. Am. J. Med. Genet. 63, 461-467. doi: 10.1002/(sici)10968628(19960614)63:3<461::aid-ajmg9>3.0.co;2-u

Xu, K., Chen, S., Xie, L., Qiu, Y., Bai, X., Liu, X. Z., et al. (2021). Local Macrophage-Related Immune Response Is Involved in Cochlear Epithelial Damage in Distinct Gjb2-Related Hereditary Deafness Models. Front. Cell Dev. Biol. 8:597769. doi: 10.3389/fcell.2020.597769

Xu, L., Qiu, X., Zhu, Z., Yi, L., and Qiu, Y. (2014). A novel mutation in COL2A1 leading to spondyloepiphyseal dysplasia congenita in a three-generation family. Eur. Spine J. 23(Suppl. 2), 271-277. doi: 10.1007/s00586-014-3292-0

Yoshida, K., Ichimiya, I., Suzuki, M., and Mogi, G. (1999). Effect of proinflammatory cytokines on cultured spiral ligament fibrocytes. Hear. Res. 137, 155-159. doi: 10.1016/s0378-5955(99)00134-3 
Zhang, J., Yang, R., Liu, Z., Hou, C., Zong, W., Zhang, A., et al. (2015). Loss of lysyl oxidase-like 3 causes cleft palate and spinal deformity in mice. Hum. Mol. Genet. 24, 6174-6185. doi: 10.1093/hmg/ddv333

Conflict of Interest: The authors declare that the research was conducted in the absence of any commercial or financial relationships that could be construed as a potential conflict of interest.
Copyright (c) 2021 Liu, Bai, Wan, Mo, Chen, Zhang and Gao. This is an openaccess article distributed under the terms of the Creative Commons Attribution License (CC BY). The use, distribution or reproduction in other forums is permitted, provided the original author(s) and the copyright owner(s) are credited and that the original publication in this journal is cited, in accordance with accepted academic practice. No use, distribution or reproduction is permitted which does not comply with these terms. 